\title{
A Comparative Study on Retrieved Images by Content Based Image Retrieval System based on Binary Tree, Color, Texture and Canny Edge Detection Approach
}

\author{
Saroj A. Shambharkar \\ Department of Information Technology \\ Kavikulguru Institute of Technology \& Science \\ Ramtek
}

\author{
Shubhangi C. Tirpude \\ Department of CSE, Shri Ramdeobaba College of \\ Engineering \& Management \\ Nagpur
}

\begin{abstract}
Content based image retrieval (CBIR) is a technique in which images are indexed by extracting their low level features or high level features or both. This paper presents performance \& comparative study of the result obtained by the two different approaches of CBIR system. The resultant retrieved images by the CBIR system is based on two approaches. Both approaches of the system using two major processes one as feature extraction and other as search and retrieval. The first approach is based on extracting the features using binary tree structure, color \& texture and the second approach is based on extracting shape features using Canny Edge Detection. In both the approaches the feature vector of the query image is compared with the feature vector of the each image present in the database. In the first approach the images are retrieved and arranged according to their matching score or rank value and in the second approach retrieved images are arranged according to their mean and correlation values.
\end{abstract}

Keywords-Binary tree structure, Content Based Image Retrieval, canny edge detection, Mean, Correlation.

\section{INTRODUCTION}

Since 1990s, the demand for information indexing and retrieval is increased because of rapid growth of available multimedia contents, that's why efforts have been done on text extraction in images and videos. The collection of images in the web are growing larger and becoming more diverse. Retrieving images from such large collections is a challenging problem in front of us. To organize and classify such large amount of images is time consuming task. Therefore it is required to design a system to organize and classify the images in a database, so that the images can be retrieve fast with little amount of time. To implement this idea, the CBIR system is introduced. It is an automated system that searches query image in an image database and retrieving the relevant images of using similarity measure between it and every image in the image data base.

It can simplify many tasks in many application areas such as biomedicine, forensics, artificial intelligence, military, education, web image searching.

\section{A. Need of CBIR System}

In recent years, there has been a growing interest in developing effective methods for content based image clustering and retrieval. This interest has been motivated by the need to efficiently manage large image databases and efficiently run image retrieval to get the best results without exhaustively searching the global database each time. This leads to huge savings in time and money, especially in fields where the bulk of working databases are image files or any kind of media whose contents cannot be described by simple keywords or short texts. With image retrieval system such as Content Based Image retrieval this limitation will not exist and system will retrieve the images as per the human can perceive, and too much responsibility on the end-user, problem of abstract needs, the queries that cannot be described at all, but tap into the visual features of images.

Most of the CBIR systems perform the search based on following:

- Keywords - This is a search in which the user poses a simple query in the form of a word or bigram. This is currently the most popular way to search images, for example, the Google and Yahoo! image search engines.

- Text - This is where the user frames a complex

- Text-phrase, sentence, question, or story about what user desires from the system.

- Image - Here, the user wishes to search for an image similar to a query image. Using an example image is perhaps the most representative way of querying a CBIR system in the absence of reliable metadata.

- Graphics-This consists of a hand-drawn or computergenerated picture, or graphics could be presented as query.

- Composite- These are methods that involve using one or more of the above mentioned that is image and keywords, or text, keyword and image for querying a system. This 
also covers interactive querying such as in relevance feedback approach [2].

From the above mentioned our CBIR system is based on color, shape and texture, binary tree.

The CBIR system is divided into following stages:

- Preprocessing: The image is first processed in order to extract the features, which describe its contents. The processing involves filtering, normalization, segmentation, and object identification. The output of this stage is a set of significant regions and objects.

- Feature Extraction: Features such as shape, texture, color, etc. are used to describe the content of the image. Image features can be classified into primitives.

\section{TYPICAL CBIR STRUCTURE}

The overall structure of a typical image retrieval system is shown in fig. 1. It consists of images, feature extraction and retrieval process. First the images are obtained from different sources. One of the images from the existing source is considered as query image and remaining images forms an image database. The feature extraction process is applied on images in the database and the query image and the feature vector is obtained. The search \& retrieval process search the query image matched with images in the database. Finally images are received and arranged according to their matching score.

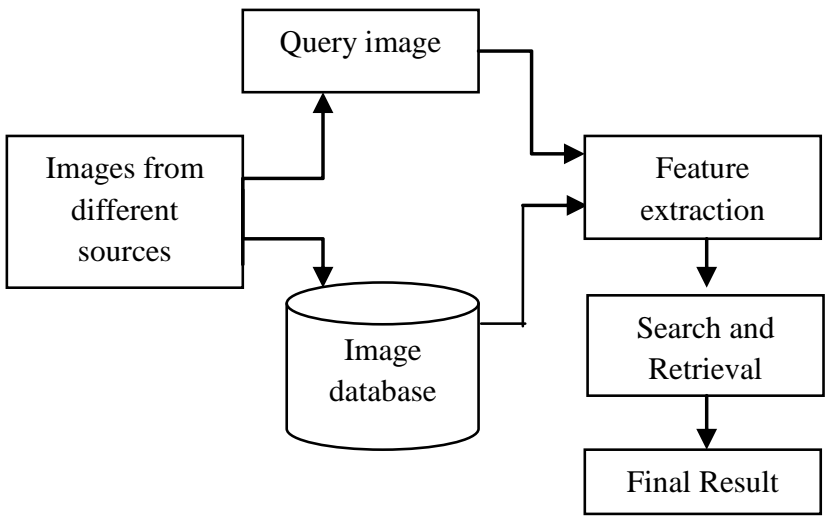

Fig. 1: Structure of CBIR

\section{QUERY PROCESSING FOR THE CBIR}

In the CBIR systems the query processing is based on the following:

- Text-Based: - Text-based query processing usually boils down to performing one or more simple keyword-based searches and then retrieving matching pictures. Processing a free text could involve parsing, processing, and understanding the query as a whole.

- Content-Based: - Content-based query processing lies at the heart of all CBIR systems. Processing of query (image or graphics) involves extraction of visual features and/or Segmentation and search in the visual feature space for similar images. An appropriate feature representation and a similarity measure to rank pictures, given a query, are essential here.

- Composite: - Composite processing may involve both content and text-based processing in varying proportions. An example of a system which supports such processing is the story picturing engine. [Joshi et al 2006b]

- Interactive-Simple: - User interaction using a single modality needs to be supported by a system. An example is a relevance-feedback based image retrieval system.

- Interactive-Composite: - The user may query using more than one modality example text and images. This can be considered as the more advanced form of query processing required for searching a query image in huge database to be performed by an image retrieval system.

Processing text-based queries involves keyword matching using simple set-theoretic operations, and therefore a response can be generated very quickly. However, in very large systems working with millions of pictures and keywords, efficient indexing methods may be required. Indexing of text has been studied in database research for decades now. Efficient indexing is critical to the building and functioning of very large text based databases and search engines. Research on efficient ways to index images by content has been largely overshadowed by research on efficient visual representation and similarity measures. Most of the methods used for visual indexing are adopted from text-indexing research [2].

\section{RELATED WORK}

There are several excellent surveys of CBIR systems. We mention here some of the more notable systems.

This paper uses a CBIR approach to retrieve building images. First, the canny edge detector is used to extract edge information from the images. Then, the Hough transform is applied to the edge map in order to reveal the linear edge distribution in the Hough transform domain. Then by using a band-wise matching (BWM) algorithm, the Hough transform domain into a number of bands and calculate the centroid of the Hough peaks in each band by partitioning. By carrying out the same aforementioned procedures on a query image and the images in the database, the similarity between the centroid of the query image and the images in the database is measured. Finally, based on the similarity measures, the CBIR system ranks the images in the database and retrieves a specified number of images with the highest rank values [3].

In this paper, the performance comparison between shape $\&$ color features in content-based image retrieval is studied. There is a great need for developing an efficient technique for finding the images. In order to find an image, image has to be represented with certain features. In this paper an efficient image retrieval technique is used which uses dynamic dominant color, and shape features of an image [4]. 
The CBIR systems can also provide an efficient medical image data retrieval from a huge content of medical database using one of the images content such as image shape. The main objective of this paper is to provide an efficient tool which is used for efficient medical image retrieval from a huge content of medical image database and which is used for further medical diagnosis purposes [5].

The paper on CBIR using the knowledge of texture, color, and binary tree structure says that in recent years, Content Based Image Retrieval (CBIR) has played an important role in many fields, such as medicine, geography, security, etc. General approaches in CBIR are based on visual attributes of images such as color, texture, shape, and layout and object [7]. Most of CBIR systems are designed to find the top $\mathrm{N}$ images that are most similar to the input query image. In this paper the approach is to combine color, texture and a customized binary partitioning tree in order to find the images similar to a specific query image. The above mentioned tree is a customized binary partitioning tree which keeps a combination of color and layout information of an image. To extract color information, two histograms of the image in HSV color space are used with 360 and 100 bins. Also a 2-levels Wavelet decomposition of image blocks is used to attain texture. The binary tree is used to maintain the image layout information [8].

\section{PROPOSED SYSTEM}

The main objective of the proposed system to provide an efficient tool for efficient image retrieval from a huge content of image database using features based on Color, Texture , Binary tree structure and Canny edge detection method and retrieve the images to identify the most similar images to the query image. The terms and methods used in both the approaches are explained below.

\section{A. Features Used by CBIR System}

Following are the features used by proposed CBIR system

\section{1) Shape}

Shape is an important and most powerful feature used for image classification, indexing and retrievals. Shape information extracted using histogram of edge detection. In this paper, the edge information in the image is obtained by using the canny edge detection. Other techniques for shape feature extraction are elementary descriptor, Fourier descriptor, template matching, Quantized descriptors and so on[5].

\section{2) Color}

Color may be one of the most straightforward features utilized by humans for visual recognition and discrimination. However, people show the natural ability of using different levels of color specificity in different contexts. For example, people would typically describe an apple as being 'red', probably implying some type of reddish hue. But in the context of describing the color of a car a person may choose to be more specific instead using the terms 'dark red' or 'maroon'. Color extraction by computer is performed without benefit of a context. Lack of knowledge also makes it difficult to cull the color information from the color distortion. The appearance of the color of real world objects is generally altered by surface texture, lighting and shading effects, and viewing conditions. The color feature is one of the most widely used visual features in image retrieval. Images characterized by color features have many advantages like robustness, effectiveness, implementation simplicity, computational simplicity, low storage requirement [6].

\section{3) Texture}

Texture features are intended to capture the granularity and repetitive patterns of surfaces within a picture. For instance, grassland, brick walls, teddy bears, and flower petals differ in texture, by smoothness as well as patterns. This feature used in domain specific image retrieval, such as in aerial imagery and medical imaging, is particularly vital due to their close relation to the underlying semantics in these cases. Texture features have long been studied in image processing, computer vision, and computer graphics [Haralick 1979], such as multi orientation filter banks [Malik and Perona 1990] and wavelet transforms [Unser 1995]. In image processing, a popular way to form texture features is by using the coefficients of a certain transform on the original pixel values, or, more sophisticatedly, by statistics computed from these coefficients. Examples of texture features using the wavelet transform and the discrete cosine transform can be found in Do and Vetterli [2002] and Li et al [2000].

\section{4) Binary Tree Structure}

Binary partition tree is a structure used to represent the regions of an image. A binary tree is used as a base for each region of each image in the database. In the binary partitioning tree leaves represents regions belonging to the initial partition. The root node corresponds to the entire image.

To construct a binary tree, the algorithm starts from an arbitrary region which considered as the first node and then selecting a neighbor region as its sibling, these nodes are added as children of their parent. This process is repeated until all regions have been added to the binary partitioning tree. To have more precise measure, each image in the database is divided into equal fixed-sized squared blocks. A distinct tree should be created for each block. For each node of the constructed binary partitioning tree, calculate the mean color and area of its corresponding region. These values i.e. mean color and mean area for all nodes are concatenated to construct a feature vector representing one block. The process of concatenating feature vectors of a block is repeated for all blocks to construct a feature vector for entire image.

\section{5) Mean}

To find out the Mean value we used the median filter which is also called sliding window spatial filter. It replaces the center value in the window with the median of all the pixel values in the window

\section{6) Correlation}

Correlation is used to determine the degree of similarity between data sets. One application of correlation is to perform template matching. The idea is to find the position where a best match exists between a small pattern or template and a set of patterns in a larger image. Similar to convolution, correlation also has a theorem that relates spatial domain processing with frequency domain processing via the Discrete 
Fourier Transform (DFT). Thus, correlation can be performed either in the spatial domain or frequency domain.

\section{Proposed Approach}

The proposed approach is described with the help of fig. 2 . Initially images from the different available sources were taken as an input to the system. These images can be preprocessed to reduce noise by filtering. In canny edge detection approach the median filtering is used. The main idea of median filter is to run through the signal entry by entry, replacing each entry with median of neighboring entries. The pattern of neighbors is called the "window", which slides, entry by entry, over the entire signal.

From the images input to the system, one of the images will be considered as query image and the remaining images forms image database. An image is a spatial representation of an object and represented by a matrix of intensity value. It is sampled at points known as pixels and represented by color intensity in RGB color model. A basic color image could be described as three layered image with each layer as Red, Green and Blue. These three colors constitute an image pixel.

Next the image feature database is created using two different approaches. First method uses the feature extraction process using the features as color, texture and binary tree structure. The second method extracts the feature shape using Canny Edge Detection (CED) algorithm. Canny edge detection out performs many of the newer algorithms that have been developed in the industry.

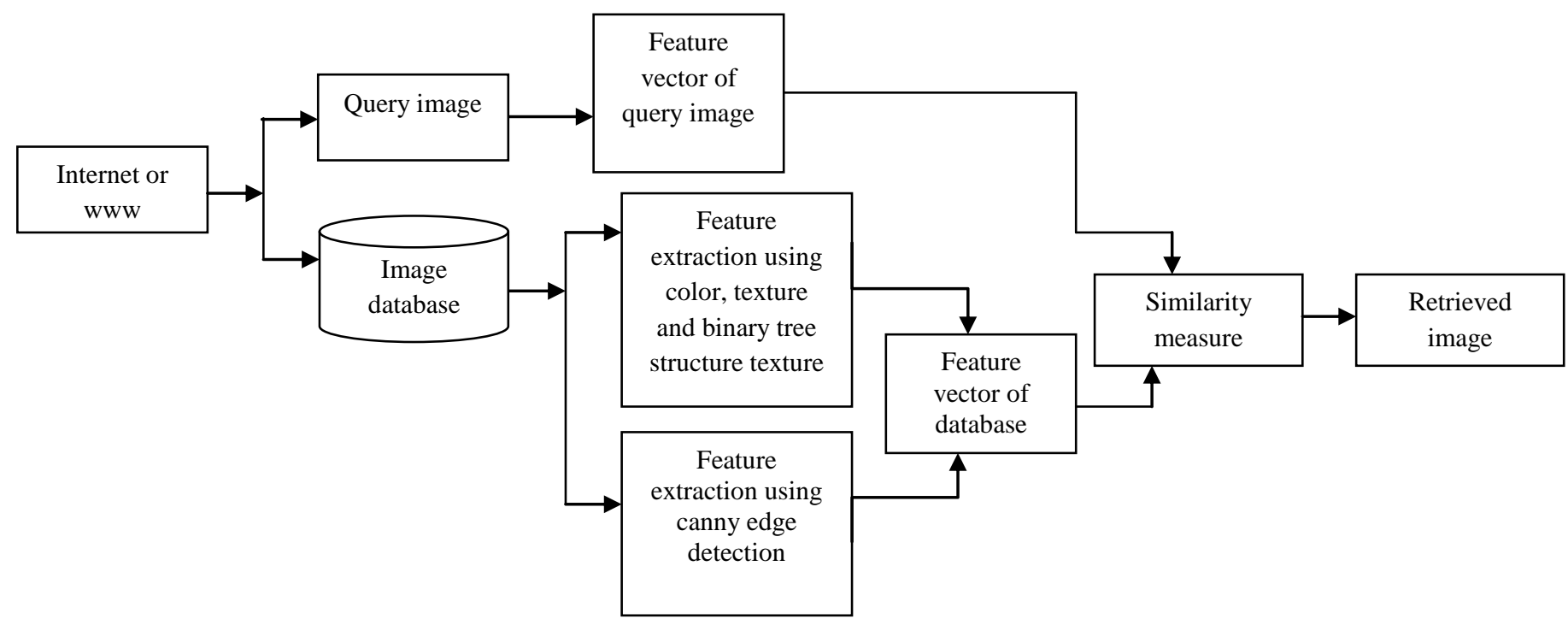

Fig. 2: Proposed CBIR Structure

The steps involved in CED algorithm for detecting edges are:

- Smoothing: Smooth the image with a two dimensional Gaussian. In most cases the computation of a two dimensional Gaussian is costly, so it is approximated by two one dimensional Gaussians.

- Finding Gradients: Take the gradient of the image this shows changes in intensity, which indicates the presence of edges. This actually gives two results, the gradient in the $\mathrm{x}$ direction and the gradient in the $\mathrm{y}$ direction.

- Non-maximal suppression: Edges will occur at points where the gradient is at a maximum. The magnitude and direction of the gradient is computed at each pixel.

- Edge Threshold: The method of threshold used by the Canny Edge Detector is referred to as "hysteresis". It makes use of both a high threshold and a low threshold.
- The feature vector of query image is also created. Next the similarity comparison technique has been carried out between feature vector of database images and the query image in both the approaches. After comparison, resulting images are indexed according to matching score value associated to each retrieved images in the first approach and in the second approach resulting images are indexed according to mean and correlation value.

\section{RESULT AND CONCLUSION}

The result of the CBIR system using two different approaches are shown in figure 3 and figure 4 which shows the retrieved images after the calculating the distance between the query image and the images from the database. In the first approach using Minkowski distance and second approach using Euclidean distance.

The result shows that retrieved images using both the approaches are quite same and if both the approaches are combined together it may increase the retrieval efficiency of 
the system. The two snapshot shows that the first two images retrieved by both the approaches are same but other images are different. To improve the result and achieve more efficient retrieval both the approaches can be combined and tested on the huge amount of images.

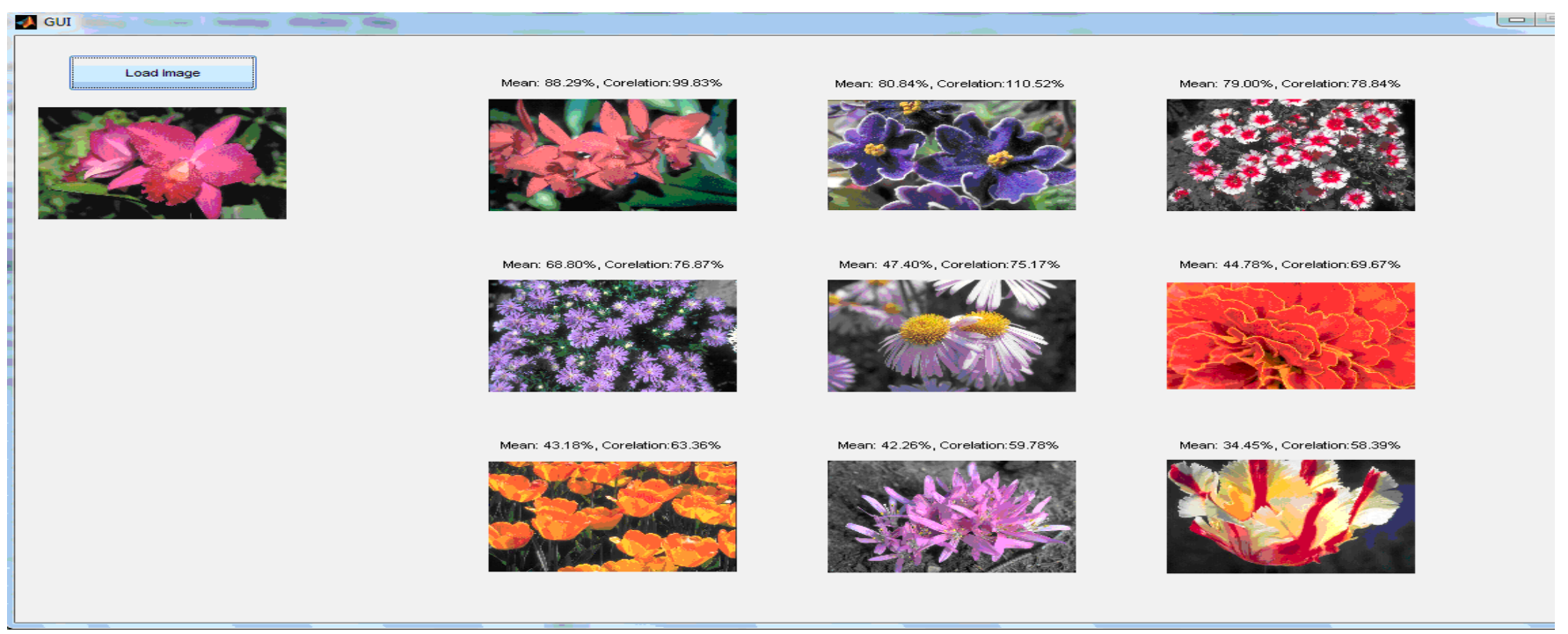

Fig. 3: Retrieved images using canny edge detection

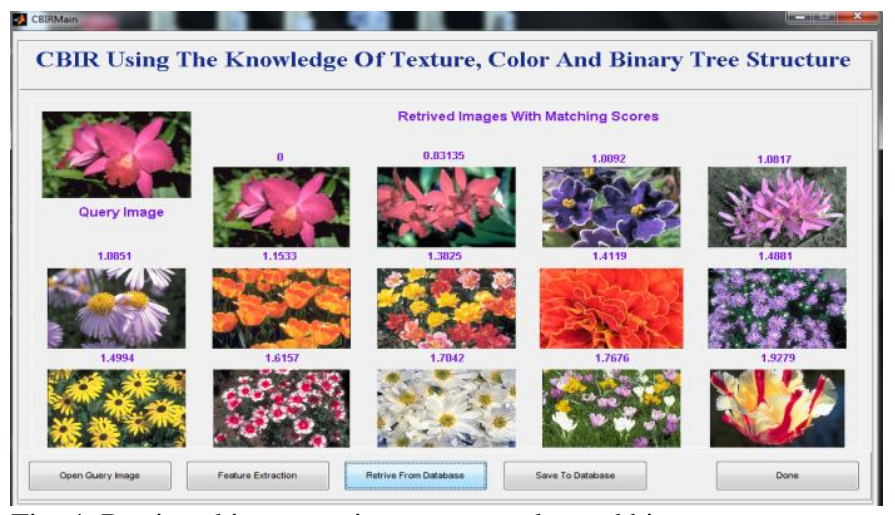

Fig. 4: Retrieved images using texture, color and binary tree structure

\section{REFERENCES}

[1] Subhankar Biswas, "A system for content Based Image Retrieval".

[2] Ritendra data, Dhiraj Joshi, Jia Li and James Z.Wang, "Image Retrieval: Ideas Influences and trends of the new Age".

[3] R F Xiang Yuan; Chang-Tsun Li; "CBIR approach to building image retrieval on linear edge distribution" IEEE International Conference 2006.

[4] Pooja Verma and Manish Mahajan, annella, "Content based image retrieval color by comparing color and shape feature" International Journal of Computer Science and Communication Vol. 2, No. 2, JulyDecember 2011, pp. 641-643. J.

[5] B. Ramamurthy, K.R. Chandran "CBMIR: shape based image retrieval using canny edge detection and $\mathrm{k}$ means clustering algorithm" International Journal of Computer Application (0975 - 8887) Volume 17- No.6, March 2011

[6] B. Ramamurthy, K.R. Chandran "CBMIR: shape based image retrieval using canny edge detection and $\mathrm{k}$ means clustering algorithm" International Journal of Computer Application (0975 - 8887) Volume 17-No.6, March 2011

[7] Ryszard S. Chora Image Feature extraction Techniques and their application for CBIR and Biometrics system.
[8] Ritendra Datta, Dhiraj Joshi, Jia Li, and James Z. Wang, Image Retrieval: Ideas, Influences, and Trends of the New Age

[9] Zahra Mansoori, Mansour Jamzad, (2009), "Content based image retrieval using the knowledge of texture, color and binary tree structure", IEEE.

[10] P.S. Hiremath and Jagadesh Pujari, "Content-Based Image Retrieval based on color, texture and shape features using image and its complement", 2007.

[11] Ritendra data, Dhiraj Joshi, Jia Li and James Z.Wang, "Image Retreival: Ideas Influences and trends of the new Age".

[12] R F Xiang Yuan; Chang-Tsun Li; "CBIR approach to building image retrieval on linear edge distribution" IEEE International Conference 2006.

[13] Pooja Verma and Manish Mahajan, annella, "Content based image retrieval color by comparing color and shape feature" International Journal of Computer Science and Communication Vol. 2, No. 2, JulyDecember 2011, pp. 641-643. J.

[14] B. Ramamurthy, K.R. Chandran "CBMIR: shape based image retrieval using canny edge detection and $\mathrm{k}$ means clustering algorithm" International Journal of Computer Application (0975 - 8887) Volume 17-No.6, March 2011

[15] B. Ramamurthy, K.R. Chandran "CBMIR: shape based image retrieval using canny edge detection and $\mathrm{k}$ means clustering algorithm" International Journal of Computer Application (0975 - 8887) Volume 17-No.6, March 2011

[16] Ryszard S. Chora Image Feature extraction Techniques and their application for CBIR and Biometrics system.

[17] Ritendra Datta, Dhiraj Joshi, Jia Li, and James Z. Wang, Image Retrieval: Ideas, Influences, and Trends of the New Age

[18] Zahra Mansoori, Mansour Jamzad, (2009), "Content based image retrieval using the knowledge of texture, color and binary tree structure", IEEE.

[19] P.S. Hiremath and Jagadesh Pujari, "Content-Based Image Retrieval based on color, texture and shape features using image and its complement", 2007. 